JULIANO BRUM SCHEFFER ${ }^{1}$

Daniel Méndez Lozano ${ }^{1}$

RENÉ FrYDMAN ${ }^{2}$

Augusto BussaB ${ }^{1}$

JOeLLE TAIEB ${ }^{3}$

RENATO FANCHIN ${ }^{4}$

\section{Artigo original}

Palavras-chave

Hormônio antimülleriano Hormônio folículo estimulante Indução da ovulação Folículo ovariano

Keywords

Anti-müllerian hormone Follicle stimulating hormone Ovulation induction Ovarian follicle

\title{
Evidência da resposta folicular ao FSH pelo hormônio antimülleriano em mulheres ovulatórias
}

\author{
Evidence of follicle responsiveness to FSH by antimuillerian \\ bormone in ovulating women
}

Resumo

OBJETIVO: examinar a hipótese de que o nível sérico do hormônio antimülleriano $(A M H)$ reflete a resposta dos folículos antrais à administração do FSH. MÉTODOS: estudo prospectivo, no qual foram incluídas 116 pacientes normo-ovulatórias inférteis submetidas à hiperestimulação ovariana controlada com agonista de GnRH e FSH. Depois de atingir a supressão pituitária e antes da administração de FSH (dia basal), o nível sérico de AMH foi mensurado. número de folículos antrais foi determinado pela ultrassonografia no dia basal lfolículos antrais precoces; 2 a 8 $\mathrm{mm}$ ) e no dia da administração do hCG (dhCG; folículos pré-ovulatórios; $\geq 16$ mm). A resposta folicular ao FSH foi determinada pela percentagem de folículos antrais precoces que atingiram os estágios pré-ovulatórios em resposta ao FSH (taxa de maturação). Foram estudadas as correlações do AMH com a idade das pacientes, número total de folículos antrais precoces e pré-ovulatórios, oócitos coletados, dose total de FSH na estimulação ovariana controlada e a taxa de maturação folicular. Para análise estatística, foram usados a regressão simples e o teste de Spearman's, com nível de significância de 5\%. RESULTADOS: o nível sérico de AMH foi positivamente correlacionado com o número de folículos antrais precoces no dia basal $(r=0,64 ; p<0,0001)$ e folículos pré-ovulatórios em dhCG $(r=0,23 ; p=0,01)$. Excepcionalmente, o nível sérico de $\mathrm{AMH}$ foi negativamente correlacionado com a taxa de maturação ( $\mathrm{r}=-0,24$; $p<0,008)$. CONCLUSÕES: o AMH atenua o desenvolvimento folicular em resposta à administração do FSH.

\section{Abstract}

PURPOSE: to test the hypothesis that the anti-müllerian hormone (AMH) serum level reflects the antral follicles' response to the administration of FSH. METHODS: prospective study, including 116 normo-ovulatory infertile patients submitted to controlled ovarian hyperstimulation with $\mathrm{GnRH}$ and FSH agonists. The $\mathrm{AMH}$ serum level was measured after reaching the pituitary suppression and before the FSH administration (basal day). The number of antral follicles was determined by ultrasonography at the basal day (precocious antral follicles; 2 to $8 \mathrm{~mm}$ ) and at the day of hCG administration (dhCG; pre-ovulatory follicles; $\geq 16 \mathrm{~mm}$ ). The follicle response to $\mathrm{FSH}$ was determined by the percentage of precocious antral follicles which reached pre-ovulatory stage in response to FSH (maturation rate). The correlation of AMH with the patients' age, the total number of precocious antral and pre-ovulatory follicles, collected oocytes, total dose of FHS in the controlled ovarian stimulation and the rate of follicular maturation was studied. For the statistical analysis, it simple regression analysis and the Spearman's test were used, at a 5\% significance level. RESULTS: The serum level of AMH was positively correlated with the number of precocious antral follicles at the basal day $(r=0.64 ; p<0.0001)$ and pre-ovulatory follicles in dhCG $(r=0.23 ; p=0.01)$. Exceptionally, the serum level of $A M H$ was negatively correlated with the maturation ratio $(r=-0.24 ; p<0.008)$. CONCLUSIONS: AMH attenuates the follicular development caused by FSH administration.

Correspondência:

Juliano Scheffer Rua Desembargador Jorge Fontana, $408,10^{\circ}$ andar - Belvedere (EP 30320-670 - Belo Horizonte (MG), Brasil Tel/Fax: (31) 3286-8171 E-mail: julianoscheffer@hotmail.com

Recebido $12 / 1 / 09$
Ambulatório de Medicina Reprodutiva do Hôpital Antoine-Béclère - Clamart, França.

' Membro da Equipe do Serviço de Medicina Reprodutiva do Hôpital Antoine-Béclère - Clamart, França.

${ }^{2}$ Chefe do Departamento de Ginecologia e Obstetrícia do Hôpital Antoine-Béclère, Clamart, França; Professor Pós-doutor da Universidade Paris XI - Clamart, França.

${ }^{3}$ Chefe do Laboratório de Bioquímica e Hormonologia do Hôpital Antoine-Béclère - Clamart, França.

${ }^{4}$ Chefe do Departamento de Medicina Reprodutiva do Hôpital Antoine-Béclère-Clamart, França; Professor Pós-doutor da Universidade Paris XI - Clamart, França.

Trabalho apresentado no 63rd Annual Meeting of American Society for Reproductive Medicine;October 13-17, 2008- Washington, D.C 


\section{Introdução}

A quantidade e a qualidade dos folículos ovarianos refletem a reserva ovariana. Esta pode ser avaliada por marcadores indiretos séricos ${ }^{1-3}$ ou ultrassonográficos ${ }^{4,5}$. Dentre os marcadores sanguíneos, o hormônio antimülleriano (AMH) é atualmente um promissor biomarcador do status ovariano ${ }^{1}$, além de predizer resultados da fertilização in vitro ${ }^{6-8}$.

Em mulheres adultas, a função fisiológica do $\mathrm{AMH}$, um peptídio exclusivamente produzido pelas células da granulosa dos folículos ovarianos, ainda não é bem estabelecida. Pesquisas experimentais sugerem o envolvimento do AMH no controle do crescimento folicular em ratos? Em mulheres, algumas observações sugerem a hipótese de que o $\mathrm{AMH}$ atenua a resposta folicular ao hormônio estimulante folicular $(\mathrm{FSH})^{10}$. Uma delas é a parada de crescimento dos folículos antrais precoces observada em pacientes oligo-anovulatórias com síndrome dos ovários policísticos (SOP). Estas apresentam excesso de folículos antrais que estão associados ao aumento periférico do nível do $A M H^{11,12}$ e no fluido folicular ${ }^{13}$, suportando a hipótese do $\mathrm{AMH}$ na anormalidade da resposta folicular ao $\mathrm{FSH}^{14}$, clinicamente notável pelos ciclos oligo-anovulatórios.

Durante o ciclo menstrual, o AMH apresenta leves flutuações sanguíneas, não interferindo na interpretação da reserva ovariana ${ }^{15-17}$, ao contrário da dosagem do $\mathrm{FSH}^{18}$.

Em mulheres normo-ovulatórias, a influência do AMH na resposta folicular, caso exista, parece ser menos notável. Além disso, nessas mulheres, o nível sérico do AMH tem sido positivamente associado à duração da resposta ovariana à hiperestimulação ovariana controlada $(\mathrm{HOC})^{19-21}$. Entretanto, esses dados clínicos não contradizem a hipótese de que o $\mathrm{AMH}$ atenue a resposta dos folículos antrais ao FSH. Realmente, a intensidade da resposta ovariana à HOC é determinada por duas variáveis. Uma delas é o tamanho do reserva folicular prétratamento. Mulheres com mais folículos antrais tendem a produzir mais folículos maturos e ovos fertilizados em resposta à administração do $\mathrm{FSH}^{22}$. A segunda variável é a capacidade inerente dos folículos antrais em atingir o estágio pré-ovulatório em resposta ao $\mathrm{FSH}^{23,24}$. Somente folículos com adequada sensibilidade às gonadotrofinas $\mathrm{e}$ competência ao desenvolvimento podem atingir a maturação final. Então, a relação quantitativa entre o AMH e a resposta ovariana à HOC pode meramente resultar em uma correlação positiva que existe entre o nível periférico de AMH e o número de folículo antral precoce nos três primeiros dias do ciclo menstrual ${ }^{25-28}$, e não avaliar o taxa de maturação dos folículos antrais precoces ao FSH.

Portanto, para averiguar a hipótese de que o $\mathrm{AMH}$ interfere na resposta folicular ao FSH em mulheres normo-ovulatórias, calculamos a razão entre o número de folículos que atingiram a maturação em resposta ao
FSH sobre a reserva folicular inicial ("taxa de maturação folicular") e, assim, avaliamos a função do AMH na resposta folicular à HOC.

\section{Métodos}

Foi realizado um estudo prospectivo no Ambulatório de Medicina Reprodutiva do Hôpital Antoine-Béclère, situado em Clamart, França. Avaliamos mulheres inférteis, com idade entre 21 e 41 anos, nível superior completo e que apresentavam os seguintes critérios para serem incluídas no estudo: ambos os ovários presentes, sem anormalidades morfológicas e adequadamente visualizados pela ultrassonografia transvaginal; ciclos menstruais regulares com duração entre 25 e 35 dias; nenhuma doença em curso ou passada que afetasse os ovários ou a secreção, excreção e clearance de gonadotrofinas e esteroides sexuais; sem sinal clínico de hiperandrogenismo; índice de massa corpórea entre 18 e $25 \mathrm{~kg} / \mathrm{m}^{2}$; número total de folículos antrais precoces (2 a $8 \mathrm{~mm}$ de diâmetro) em ambos os ovários menor que 24; sem evidência de endometriose; sem evidência de ovários policísticos (critérios de Rotterdam). Foram, então, incluídas 116 mulheres inférteis. A infertilidade era devida à anormalidade espermática (54\%), anormalidade nas tubas (29\%) ou inexplicada (17\%). O consentimento informado foi obtido de todas as mulheres, e a investigação foi aprovada pela Comissão de Ética da nossa instituição.

\section{Protocolo de HOC}

Todas as mulheres receberam agonista do hormônio liberador de gonadotrofinas $(\mathrm{GnRH})$, triptorelina (3mg/dia im, Decapeptyl, Beaufour Ipsen Pharma, Paris, France), no primeiro dia do ciclo. Três semanas depois da aplicação do agonista, a dessensibilização da hipófise foi confirmada pela detecção de baixo nível sérico de estradiol $\left(E_{2}\right)$ e gonadotrofinas. As pacientes também foram submetidas ao exame ultrassonográfico convencional para excluir cistos ovarianos e também para verificar se a espessura endometrial era $<5 \mathrm{~mm}$. Terapia com FSH recombinante (Gonal-F, Serono Pharmaceuticals, Boulogne, France) foi iniciada com a dose de $225 \mathrm{UI} /$ dia e continuada até o dia da administração do hCG $(10,000$ UI, im, Gonadotrophine Chorionique Endo ${ }^{\circledR}$, Organon Pharmaceuticals, Saint-Denis, France). Depois do sexto dia de terapia de FSH recombinante, a dose diária de FSH foi ajustada de acordo com o número dos folículos em crescimento. Administração de hCG foi realizada quando pelo menos quatro folículos eram superiores a $16 \mathrm{~mm}$ de diâmetro, e o nível de $\mathrm{E}_{2}$ por folículo maturo ( $\geq 16 \mathrm{~mm}$ de diâmetro) foi $>200 \mathrm{pg} / \mathrm{Ml}^{29-32}$. Mulheres que não apresentaram esses critérios tiveram o seu ciclo de estimulação cancelado. Os oócitos foram obtidos 36 horas depois da administração do hCG pela aspiração folicular 
guiada pelo ultrassom transvaginal. Todos os embriões foram transferidos dois dias depois da punção folicular utilizando o cateter Frydman (CCD Laboratories, Paris, France). A fase lútea foi auxiliada com progesterona micronizada $\left(\mathrm{P}_{4}\right)(600 \mathrm{mg} / \mathrm{dia}$, Estima, Effik Pharmaceuticals, Bièvres, France) administrada diariamente por via vaginal, iniciada na noite da transferência embrionária.

\section{Avaliação hormonal e folicular}

Para o propósito desse estudo, consideramos a amostra de sangue como dia basal no dia em que a dessensibilização da hipófise foi confirmada e dia hCG (dhCG) no dia da administração do hCG . Todas as amostras de sangue foram obtidas por punções venosas, e o plasma foi separado e congelado a $-20^{\circ} \mathrm{C}$ para subsequente análise. No dia basal, o nível sérico de $\mathrm{AMH}$ foi determinado usando método enzimático de imunoquimioluminescência (ELISA referência A16507; Immunotech Beckman Coulter Laboratories, Villepinte, France). Intra e intercoeficiente de variação (CV) foram $<6$ e $<10 \%$, respectivamente; o limite mínimo de detecção foi 0,13 ng/mL, e o máximo, $21 \mathrm{ng} / \mathrm{mL}$ para AMH. No dia basal e dhCG, o nível sérico de $\mathrm{E}_{2}$ e $\mathrm{P}_{4}$ foram determinados por sistema de multianálise automatizado, usando a técnica

Tabela 1 - Correlação entre o hormônio antimülleriano e algumas variáveis

\begin{tabular}{l|ccc}
\hline Variáveis & Média e desvio padrão & $r$ & Valor de p \\
\hline Idade & $33,0 \pm 0,37$ anos & $-0,21$ & $<0,01$ \\
Número de folículos antrais precoces & $17,0 \pm 0,25$ & 0,64 & $<0,0001$ \\
Número de folículos pré-ovulatórios & $8,0 \pm 0,25$ & 0,23 & $<0,01$ \\
Número de oócítos coletados & $11 \pm 0,40$ & 0,38 & $<0,0001$ \\
\hline Taxa de maturação folicular & & $-0,29$ & $<0,007$ \\
\hline
\end{tabular}

Taxa de maturação folicular=razão entre número de folículos pré-ovulatórios e antrais precoces.

$r=$ coeficiente de correlação (teste de Spearman); $p<0,05=$ significância estatística.

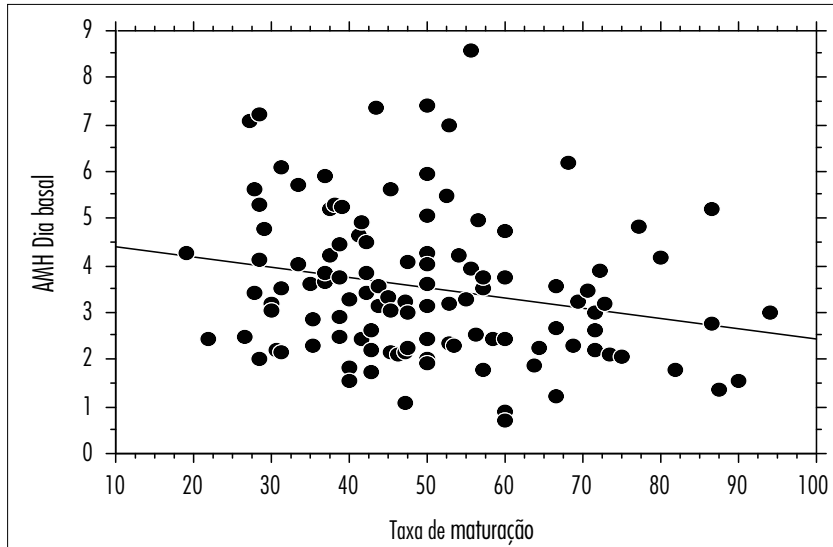

Taxa de maturação folicular=razão entre número de folículos pré-ovulatórios e antrais precoces.

dhCG=dia da administração da gonadotrofina coriônica; $r=$ coeficiente de correlação (teste de Spearman); $p<0,05=$ significância estatística.

Figura 1 - Relação entre a taxa de maturação dos folículos no dhCG e o nível sérico de hormônio antimülleriano (AMH) no dia basal (r=-0,247; $\mathrm{p}=0,008)$. de quimioluminescência (Advia-Centaur, Bayer Diagnostics, Puteaux, France). Para $\mathrm{E}_{2}$, o limite mínimo de detecção foi $15 \mathrm{pg} / \mathrm{mL}$, e o máximo, $1.000 \mathrm{pg} / \mathrm{mL}$; e intra e inter-CV foram 8 e $9 \%$, respectivamente. Para $\mathrm{P}_{4}$, o limite mínimo de detecção foi $0,1 \mathrm{ng} / \mathrm{mL}$, e o máximo, de $60 \mathrm{ng} / \mathrm{mL}$; e intra e inter-CV foram 8 e $9 \%$, respectivamente.

No dia basal e dhCG, o exame ultrassonográfico dos ovários foi realizado usando a sonda multifrequência transvaginal 5,0 a 9,0 MHz (Voluson 730 Expert, General Electric Medical Systems, Paris, France), com o objetivo de avaliar o número e o tamanho dos folículos antrais. Determinamos, no dia basal, o número e o diâmetro médio de todos os folículos entre 2 e $8 \mathrm{~mm}$ de ambos ovários e dhCG, o número e o diâmetro médio de todos os folículos entre 12 e $22 \mathrm{~mm}$. No dhCG, folículos medindo de 16 a $22 \mathrm{~mm}$ de diâmetro foram considerados maturos ${ }^{29}$.

\section{Análise estatística}

$\mathrm{Na}$ análise estatística, a mediana e os limites míninos e máximos foram utilizados para avaliação das dosagens hormonais. A média e o desvio padrão da média foram utilizadas para avaliação dos outros parâmetros, como idade, duração do ciclo menstrual e dos dias de estimulação ovariana, dose total de FSH recombinante, números de folículos antrais precoces, pré-ovulatórios, oócitos coletados e embriões transferidos. A taxa de maturação folicular foi obtida pela razão entre o número de folículos medindo entre 16 e $22 \mathrm{~mm}$ de diâmetro no dhCG e o número de folículos entre 2 e $8 \mathrm{~mm}$ de diâmetro no dia basal. A relação entre duas variáveis foi avaliada pela correlação e pel regressão simples. O teste de Spearman foi usado para determinar se os coeficientes de correlação(r) foram significativamente diferentes de zero. Consideraram-se como significantes as correlações entre as variáveis que obtivessem um valor de $\mathrm{p}<0,05$.

\section{Resultados}

No dia basal, a mediana (limites mínino e máximo) do $\mathrm{AMH}, \mathrm{E}_{2}$ e $\mathrm{P}_{4}$ sérico foram 3,53 $(0,71$ a 8,59$) \mathrm{ng} / \mathrm{mL}$, 31 (30 a 50) pg/mL e $0,25(0,1$ a 1,6$) \mathrm{ng} / \mathrm{mL}$, e o número médio de folículos antrais precoces foi $17 \pm 0,25$. O nível sérico do $\mathrm{AMH}$ mostrou correlação negativa com a idade $(\mathrm{r}=-0.21 ; \mathrm{p}<0,01)$ e correlação positiva com o número de folículos antrais precoces $(r=0,64 ; \mathrm{p}<0,0001)$, e folículos pré-ovulatórios no dhCG $(r=0,23 ; \mathrm{p}<0,01)$ e oócitos coletados $(\mathrm{r}=0,38 ; \mathrm{p}<0.0001)$ (Tabela 1$)$.

No dhCG, a média do número de folículos maturos (16 a $22 \mathrm{~mm}$ de diâmetro) foi $8 \pm 0,25$. A relação entre a taxa de maturação dos folículos no dhCG e o nível do AMH está demonstrada na Figura 1. O nível sérico do AMH foi negativamente correlacionado com a taxa de maturação $(r=-0,24 ; p<0,008)$. 
A média de idade das mulheres e a duração do ciclo menstrual foram $33,0 \pm 0,37$ anos e $28,7 \pm 0,17$ dias, respectivamente. A HOC durou $12 \pm 0,13$ dias e necessitou de 2,981 \pm 74 UI de FSH recombinante. Observamos uma relação negativa entre a necessidade de $\mathrm{FSH}$ recombinate para HOC e o nível sérico de AMH no dia basal $(r=-0,29$; $\mathrm{p}<0,007)$. Em adição, o nível sérico do AMH no dia basal foi comparável entre as causas de infertilidade. Os números totais médios de oócitos coletados e embriões transferidos foram $11 \pm 0,4$, e $2,2 \pm 0,05$, respectivamente.

\section{Discussão}

O presente estudo mostrou que a percentagem de folículos sensíveis ao FSH que efetivamente respondem ao tratamento exógeno de FSH está reduzida em mulheres normo-ovulatórias com ovários multifoliculares e altos níveis de AMH.

Neste estudo, a dosagem do AMH foi realizada após a dessensibilização hipofisária, no dia basal, por ser o dia em que a paciente iria realizar a ultrassonografia endovaginal e a dosagem sérica do estradiol e FSH. Os trabalhos recentes demonstram pequenas flutuações não significativas na dosagem sérica do AMH durante o ciclo menstrual ${ }^{15-17}$, após contraceptivos orais e vaginais ${ }^{33}$ e após a administração de análogos de $\mathrm{GnRH}^{34,35}$, não interferindo, assim, em nossa conclusão sobre o papel do $\mathrm{AMH}$ em relação à sensibilidade ao $\mathrm{FSH}$.

Apesar de não ser o objetivo principal deste estudo, realizou-se a dosagem da progesterona e do estradiol no dhCG, devido ao controle da preparação endometrial e do crescimento folicular ${ }^{36}$.

O papel do AMH na foliculogênese ovariana ainda não está bem esclarecido. Em estudo em ratos, especificamente em cultura de células da granulosa, o AMH exógeno inibe a síntese e diminui a atividade da $\mathrm{P} 450$ aromatase, sugerindo que AMH apresenta um fator parácrino e um feedback negativo no desenvolvimento folicular ${ }^{37} \mathrm{e}$ na proliferação das células da granulosa luteinizadas ${ }^{38}$. Além da redução da atividade da aromatase, o AMH parece reduzir também o número de receptores de $\mathrm{LH}$ nas células da granulosa quando estimuladas pelo $\mathrm{FSH}^{39}$.

$\mathrm{O} A M H$ e seus receptores estão altamente expressos nas células da granulosa, principalmente nos folículos pré-antrais e antrais precoces, tendo função importante no recrutamento e no desenvolvimento foliculares. Este biomarcador exerce feedback negativo no recrutamento folicular, na atresia folicular, e apresenta papel determinante na sensibilidade dos folículos ovarianos ao $\mathrm{FSH}^{9,40}$, sugerido no presente estudo.

$\mathrm{O}$ mecanismo da interação entre $\mathrm{AMH}$ e a sensibilidade do folículo ao FSH ainda não está totalmente esclarecido. Pode envolver efeitos do AMH na expressão dos receptores do FSH. A redução da expressão dos receptores do FSH pode alterar a sensibilidade do folículo ao FSH, como demonstrado em estudos de bovinos ${ }^{41}$. Seguindo este mesmo raciocínio, vários trabalhos têm demonstrado que o $\mathrm{AMH}$ inibe o segundo mensageiro do $\mathrm{FSH}, \mathrm{AMPc}^{38,42,43}$.

Em mulheres com SOP, o AMH sérico está fortemente aumentado. Esse aumento é reflexo tanto do aumento da produção deste hormônio por célula da granulosa, como também pelo número aumentado de folículos nos ovários. Este nível sérico aumentado do AMH pode explicar, em parte, a refratariedade da diferenciação folicular induzida pelo FSH e a diminuição do crescimento e do desenvolvimento folicular nessas pacientes ${ }^{44,45}$.

Apesar de estas evidências e de nossos resultados demonstrarem o fator inibitório do AMH no desenvolvimento folicular, a ação do AMH na atividade ovariana parece ser complexa e ainda necessita de maiores investigações para confirmar nossos resultados.

Nosso estudo demonstrou que a percentagem de folículos sensíveis ao FSH que efetivamente respondem ao tratamento com FSH está reduzido em mulheres normoovulatórias com ovários multifoliculares e alto nível de $\mathrm{AMH}$, fato que suporta a hipótese de que o $\mathrm{AMH}$ atenua o desenvolvimento folicular ao FSH.

\section{Referências}

1. Scheffer JB, Lozano DM, Frydman R, Fanchin R. Relação entre os níveis séricos do hormônio anti-Mulleriano, inibina $B$, estradiol e hormônio folículo estimulante no terceiro dia e o status folicular ovariano. Rev Bras Ginecol Obstet. 2007;29(4):186-91.

2. Fiçicioglu C, Kutlu T, Baglam E, Bakacak Z. Early follicular antimüllerian hormone as an indicator of ovarian reserve. Fertil Steril. 2006;85(3):592-6.

3. Sun W, Stegmann BJ, Henne M, Catherino WH, Segars JH. A new approach to ovarian reserve testing. Fertil Steril. 2008;90(6):2196. 202.
4. Hendriks DJ, Kwee J, Mol BW, te Velde ER, Broekmans FJ. Ultrasonography as a tool for the prediction of outcome in IVF patients: a comparative meta-analysis of ovarian volume and antral follicle count. Fertil Steril. 2007;87(4):764-75.

5. Broekmans FJ, Kwee J, Hendriks DJ, Mol BW, Lambalk CB. A systematic review of tests predicting ovarian reserve and IVF outcome. Hum Reprod Update. 2006; 12(6):685-718.

6. Broer SL, Mol BW, Hendriks D, Broekmans FJ. The role of antimullerian hormone in prediction of outcome after IVF: comparison with the antral follicle count. Fertil Steril. 2009;91(3):705-14. 
7. Takahashi C, Fujito A, Kazuka M, Sugiyama R, Ito H, Isaka K. Anti-Müllerian hormone substance from follicular fluid is positively associated with success in oocyte fertilization during in vitro fertilization. Fertil Steril. 2008;89(3):586-91.

8. Lee $\mathrm{TH}$, Liu $\mathrm{CH}$, Huang CC, Wu YL, Shih YT, Ho HN, et al. Serum anti-Müllerian hormone and estradiol levels as predictors of ovarian hyperstimulation syndrome in assisted reproduction technology cycles. Hum Reprod. 2008;23(1):160-7.

9. Durlinger AL, Gruijters $M$, Kramer $P$, Karels B, Ingraham HA, Nachtigal MW, et al. Anti-Müllerian hormone inhibits initiation of primordial follicle growth in the mouse ovary. Endocrinology. 2002; 143(3):1076-84

10. Carlsson IB, Scott JE, Visser JA, Ritvos O, Themmen AP, Hovatta $O$. Anti-Müllerian hormone inhibits initiation of growth of human primordial ovarian follicles in vitro. Hum Reprod. 2006;21 (9):2223-7.

11. Fallat ME, Siow Y, Marra M, Cook C, Carrillo A. Müllerianinhibiting substance in follicular fluid and serum: a comparison of patients with tubal factor infertility, polycystic ovary syndrome, and endometriosis. Fertil Steril. 1997;67(5):962-5.

12. Pigny $P$, Merlen E, Robert $Y$, Cortet-Rudelli $C$, Decanter $C$, Jonard $S$, et al. Elevated serum level of anti-mullerian hormone in patients with polycystic ovary syndrome: relationship to the ovarian follicle excess and to the follicular arrest. J Clin Endocrinol Metab. 2003;88(12):5957-62.

13. Das M, Gillott DJ, Saridogan E, Diahanbakhch O. Anti-Mullerian hormone is increased in follicular fluid from unstimulated ovaries in women with polycystic ovary syndrome. Hum Reprod. 2008;23(9):2122-6.

14. Jonard S, Dewailly D. The follicular excess in polycystic ovaries, due to intra-ovarian hyperandrogenism, may be the main culprit for the follicular arrest. Hum Reprod Update. 2004; 10(2): 107-17

15. La Marca A, Giulini S, Tirelli A, Bertucci E, Marsella T, Xella S, et al. Anti-Müllerian hormone measurement on any day of the menstrual cycle strongly predicts ovarian response in assisted reproductive technology. Hum Reprod. 2007;22(3):766-71.

16. La Marca A, Stabile G, Artenisio AC, Volpe A. Serum anti-Mullerian hormone throughout the human menstrual cycle. Hum Reprod. 2006;21(12):3103-7.

17. Tsepelidis S, Devreker F, Demeestere I, Flahaut A, Gervy Ch, Englert $Y$. Stable serum levels of anti-Müllerian hormone during the menstrual cycle: a prospective study in normo-ovulatory women. Hum Reprod. 2007;22(7): 1837-40

18. Barad DH, Weghofer A, Gleicher N. Comparing anti-Müllerian hormone (AMH) and follicle-stimulating hormone (FSH) as predictors of ovarian function. Fertil Steril. In press 2009.

19. Seifer DB, MacLaughlin DT, Christian BP, Feng B, Shelden RM. Early follicular serum müllerian-inhibiting substance levels are associated with ovarian response during assisted reproductive technology cycles. Fertil Steril. 2002;77(3):468-71.

20. Hazout A, Bouchard P, Seifer DB, Aussage P, Junca AM, Cohen-Bacrie P. Serum antimüllerian hormone/müllerianinhibiting substance appears to be a more discriminatory marker of assisted reproductive technology outcome than follicle-stimulating hormone, inhibin B, or estradiol. Fertil Steril. 2004;82(5):1323-9.

21. Fanchin R, Louafi N, Méndez Lozano DH, Frydman N, Frydman R, Taieb J. Per-follicle measurements indicate that anti-müllerian hormone secretion is modulated by the extent of follicular development and luteinization and may reflect qualitatively the ovarian follicular status. Fertil Steril. 2005;84(1):167-73
22. Melo MA, Garrido N, Alvarez C, Bellver J, Meseguer M, Pellicer A, et al. Antral follicle count (AFC) can be used in the prediction of ovarian response but cannot predict the oocyte/embryo quality or the in vitro fertilization outcome in an egg donation program. Fertil Steril. 2009;91(1): 148-56.

23. Mendoza C, Ruiz-Requena E, Ortega E, Cremades N, Martinez F, Bernabeu R, et al. Follicular fluid markers of oocyte developmental potential. Hum Reprod. 2002;17(4):1017-22.

24. Appasamy M, Jauniaux E, Serhal P, Al-Qahtani A, Groome NP, Muttukrishna S. Evaluation of the relationship between follicular fluid oxidative stress, ovarian hormones, and response to gonadotropin stimulation. Fertil Steril. 2008;89(4):912-21.

25. van Rooij IA, Broekmans FJ, te Velde ER, Fauser BC, Bancsi LF, de Jong $\mathrm{FH}$, et al. Serum anti-Müllerian hormone levels: a novel measure of ovarian reserve. Hum Reprod. 2002;17(12):3065-71.

26. de Vet A, Laven JS, de Jong FH, Themmen AP, Fauser BC. Antimüllerian hormone serum levels: a putative marker for ovarian aging. Fertil Steril. 2002;77(2):357-62

27. Fanchin $R$, Schonaver LM, Righini C, Frydman N, Frydman R, Taieb J. Serum anti-Müllerian hormone dynamics during controlled ovarian hyperstimulation. Hum Reprod. 2003; 18(2):328-3.

28. Fanchin R, Schonäuer LM, Righini C, Guibourdenche J, Frydman $\mathrm{R}$, Taieb J. Serum anti-Müllerian hormone is more strongly related to ovarian follicular status than serum inhibin $B$, estradiol, FSH and LH on day 3. Hum Reprod. 2003;18(2):323-7.

29. Dubey AK, Wang HA, Duffy P, Penzias AS. The correlation between follicular measurements, oocyte morphology, and fertilization rates in an in vitro fertilization program. Fertil Steril. 1995;64(4):787-90.

30. Miller KF, Goldberg JM, Falcone T. Follicle size and implantation of embryos from in vitro fertilization. Obstet Gynecol. 1996;8814 Pt 1):583-6

31. Ectors FJ, Vanderzwalmen P, Van Hoeck J, Nijs M, Verhaegen G, Delvigne $A$, et al. Relationship of human follicular diameter with oocyte fertilization and development after in-vitro fertilization or intracytoplasmic sperm injection. Hum Reprod. 1997;12(9):2002-5.

32. Bergh C, Broden H, Lundin K, Hamberger L. Comparison of fertilization, cleavage and pregnancy rates of oocytes from large and small follicles. Hum Reprod. 1998;13(7):1912-5.

33. Streuli I, Fraisse T, Pillet $C$, Ibecheole V, Bischof $P$, de Ziegler D. Serum antimüllerian hormone levels remain stable throughout the menstrual cycle and after oral or vaginal administration of synthetic sex steroids. Fertil Steril. 2008;90(2):395-400.

34. Lee JR, Kim SH, Kim SM, Jee BC, Ku SY, Suh CS, et al. Follicular fluid anti-Müllerian hormone and inhibin $B$ concentrations: comparison between gonadotropin-releasing hormone $(\mathrm{GnRH})$ agonist and GnRH antagonist cycles. Fertil Steril. 2008;89(4):860-7.

35. Mohamed KA, Davies WA, Lashen H. Antimüllerian hormone and pituitary gland activity after prolonged down-regulation with goserelin acetate. Fertil Steril. 2006;86(5):1515-7.

36. Papanikolaou EG, Kolibianakis EM, Pozzobon C, Tank P, Tournaye $\mathrm{H}$, Bourgain $\mathrm{C}$, et al. Progesterone rise on the day of human chorionic gonadotropin administration impairs pregnancy outcome in day 3 single-embryo transfer, while has no effect on day 5 single blastocyst transfer. Fertil Steril. 2009;91(3):949-52.

37. di Clemente N, Wilson C, Faure E, Boussin L, Carmillo P, Tizard R, et al. Cloning, expression, and alternative splicing of the receptor for anti-Müllerian hormone. Mol Endocrinol. 1994;8(8):1006-20.

38. Kim JH, Seibel MM, MacLaughlin DT, Donahoe PK, Ransil BJ, Hametz PA, et al. The inhibitory effects of müllerian-inhibiting substance on epidermal growth factor induced proliferation and progesterone production of human granulosa-luteal cells. J Clin Endocrinol Metab. 1992;75(3):911-7. 
39. Josso N, di Clemente N, Govédard L. Anti-Müllerian hormone and its receptors. Mol Cell Endocrinol. 2001;179(1-2): 25-32.

40. Durlinger AL, Gruijters M, Kramer P, Karels B, Kumar TR, Matzuk $M M$, et al. Anti-Müllerian hormone attenuates the effects of FSH on follicle development in the mouse ovary. Endocrinology. 2001;142(1 1):4891-9.

41. Bao B, Garverick HA, Smith GW, Smith MF, Salfen BE, Youngquist RS. Expression of messenger ribonucleic acid (mRNA) encoding 3 beta-hydroxysteroid dehydrogenase delta4,delta5 isomerase (3beta-HSD) during recruitment and selection of bovine ovarian follicles: identification of dominant follicles by expression of 3 beta-HSD mRNA within the granulosa cell layer. Biol Reprod. 1997;56(6): 1466-73.
42. Rouiller-Fabre V, Carmona S, Merhi RA, Cate R, Habert R, Vigier B. Effect of anti-Mullerian hormone on Sertoli and Leydig cell functions in fetal and immature rats. Endocrinology. 1998;139(3):1213-20.

43. Teixeira J, Fynn-Thompson E, Payne AH, Donahoe PK. Müllerianinhibiting substance regulates androgen synthesis at the transcriptional level. Endocrinology. 1999; 140(10):4732-8.

44. Coffler MS, Patel K, Dahan MH, Malcom PJ, Kawashima T, Deutsch $\mathrm{R}$, et al. Evidence for abnormal granulosa cell responsiveness to follicle-stimulating hormone in women with polycystic ovary syndrome. J Clin Endocrinol Metab. 2003;88(4):1742-7.

45. Wang JG, Nakhuda GS, Guarnaccia MM, Sauer MV, Lobo RA. Müllerian inhibiting substance and disrupted folliculogenesis in polycystic ovary syndrome. Am J Obstet Gynecol. 2007;196(1):77.e1-5. 